\title{
String Theory and Nonrelativistic Gravity
}

\section{Eric A. Bergshoeff*,}

Van Swinderen Institute

9747 AG Groningen, Netherlands

E-mail: E.A.Bergshoefferug.nl

\section{Ceyda Şimşek}

Van Swinderen Institute

9747 AG Groningen, Netherlands

E-mail: c.simsek@rug.nl

We identify the proper geometry underlying non-relativistic string theory as 'String NewtonCartan Gravity'. It has the distinguishing feature that one-dimensional foliation in Newton-Cartan geometry is replaced by a two-dimensional foliation on the target space. We discuss some basic properties of the string Newton-Cartan space-time, in particular it's behaviour under T-duality transformations. This leads to interesting non-relativistic conformal field theories at the boundary that have applications in condensed matter physics.

Corfu Summer Institute 2018 "School and Workshops on Elementary Particle Physics and Gravity" (CORFU2018)

31 August - 28 September, 2018

Corfu, Greece

${ }^{*}$ Speaker. 


\section{Introduction}

Assuming small spacetime curvature, the low-energy limit of superstring theory is described by a supergravity theory. Using this connection our knowledge about supergravity has teached us a lot about some basic properties of superstring theory such as T-duality, D-branes, exotic branes etc. Via it's embedding into string theory, supergravity in an AdS background has an intriguing holographic relation with a conformal field theory in one dimension lower. This latter relationship has also been investigated at the non-relativistic level by considering background geometries in the bulk that have non-relativistic isometries such as Lifshitz or Schrödinger symmetries. This leads to non-relativistic conformal field theories at the boundary that have applications in condensed matter physics. ${ }^{1}$

A different and less well studied corner of non-relativistic holography occurs when the gravity in the bulk itself becomes non-relativistic. It has been argued that the relevant non-relativistic gravity is an extension of Newton-Cartan (NC) gravity, i.e. the frame-independent formulation of Newtonian gravity. This new kind of holography leads to non-relativistic CFTs at the boundary with a different set of symmetries such as the (infinite-dimensional) Galilean conformal symmetries [1]. We are especially interested in the possibility that the non-relativist gravity theory in the bulk is connected to a non-relativistic string theory in the same way that general relativitv is related to relativistic string theory. This non-relativistic string theory could then perhaps be used as at starting point by itself, independent of the relativistic string theory, to study non-relativistic holography.

Independent of holography, it was realized in the condensed matter community that reparametrization invariance is not only relevant to gravity but also plays a crucial role in Effective Field Theories (EFTs), where one writes effective Lagrangians in an expansion organized according to the number of derivatives. Coupling an EFT to an arbitrary gravitational background enables one to study generic features of this EFT in an arbitrary frame. The application of non-relativistic gravity in this new context was pioneered by Son and coworkers [2]. The detailed understanding of how to couple a non-relativistic EFT to a general NC gravitational background can be obtained systematically by starting from a relativistic EFT in a general gravitational background that includes an auxiliary zero-flux vector field [3]. By taking a special limit of this EFT in a frame-independent way, one automatically obtains a non-relativistic EFT in a Newton-Cartan background.

This talk is organized as follows. In the first part we recall how the kinematics of NC gravity follows from gauging a specific non-relativistic algebra. We extend this analysis to String NC gravity. In the second part we discuss the non-relativistic string theory that naturally couples to the String NC geometry. In particular, we discuss the T-duality properties of this new kind of geometry. At the end we give our conclusions.

\section{Newton-Cartan Gravity}

It is well-known that the symmetries connecting non-relativistic inertial frames are given by the following set of Galilei symmetries:

\footnotetext{
${ }^{1}$ It has been found that the non-relativistic conformal field theories at the boundary couple to a Newton-Cartan geometry with twistless torsion [13].
} 

- time translation:
$\delta t=\xi^{0}$,
- space translations :
$\delta x^{i}=\xi^{i}$
$i=1,2,3$,
- spatial rotations :
$\delta x^{i}=\lambda^{i}{ }_{j} x^{j}$
- Galilean boosts :
$\delta x^{i}=\lambda^{i} t$

Here $\xi^{0}, \xi^{i}, \lambda^{i}{ }_{j}$ and $\lambda^{i}$ are the constant parameters associated with the time translation, space translations, spatial rotations and Galilean boosts, respectively. These symmetries are identical to the Poincaré symmetries except for the Galilean boosts which differ from the Lorentzian boosts in the sense that under Galilean boosts the spatial coordinates $x^{i}$ transform to the absolute time coordinate $t$, but $t$ does not transform back into the spatial coordinates $x^{i}$.

It turns out that gauging the Galilei algebra associated with the above symmetries is not sufficient to obtain NC gravity. One way to see this is to realize that the spin-connection fields associated with the spatial rotations and boost symmetries are dependent fields in General Relativity and one expects this to be the same in NC gravity. In General Relativity this is obtained by setting the torsion equal to zero which yields the 24 so-called conventional constraints needed to solve the 24 spin-connection fields associated with the Lorentz transformations. The problem with the Galilei algebra is that the curvature of time translations does not contain a spin-connection field and hence cannot be used for obtaining a conventional constraint. Therefore, there are not enough conventional constraints to solve for all spin-connections. One way to circumvent this issue is to work with a centrally extended Galilei algebra, called the Bargmann algebra. It turns out that the curvature corresponding to the central charge transformations does contain the boost spin-connection fields. By setting this curvature to zero one acquires the 6 missing conventional constraints. Together with the 18 conventional constraints that follow from setting the curvature corresponding to the spatial translations equal to zero, one is now able to solve for the 12 spatial rotation connection fields $\Omega_{\mu}{ }^{a b}(\mu=0,1,2,3 ; a=1,2,3)$ and the 12 Galilean boost connection fields $\Omega_{\mu}{ }^{a}$. The central charge introduced by the Bargmann algebra also has a physical interpretation. When coupled to matter, the U(1) central charge symmetry leads to a conserved Noether charge expressing the conservation of particle number. It also leads to the fact the the Schrödinger equation is necessarily complex providing a projective representation of the Galilei algebra.

We now consider the gauging of the Bargmann algebra [4]. Our starting point is the set of commutation relations defining the Bargmann algebra

$$
\begin{array}{ll}
{\left[J_{a b}, P_{c}\right]=-2 \delta_{c[a} P_{b]},} & {\left[J_{a b}, G_{c}\right]=-2 \delta_{c[a} G_{b]},} \\
{\left[G_{a}, H\right]=-P_{a},} & {\left[G_{a}, P_{b}\right]=-\delta_{a b} Z,}
\end{array}
$$

where $\left\{H, P_{a}, J_{a b}, G_{a}, Z\right\}$ with $a=1,2,3$ are the generators of time translation, space translations, spatial rotations, Galilean boosts and central charge transformation, respectively. In this gauging procedure we associate to every generator/symmetry a gauge field and gauge parameters that are arbitrary functions of spacetime together with the covariant curvatures, see Table 1 below. Note that we have left out the parameters corresponding to the time and space translations. Instead, we assume that all gauge fields transform as covariant vectors under general coordinate transformations 


\begin{tabular}{lllll}
\hline symmetry & generators & gauge field & parameters & curvatures \\
\hline time translation & $H$ & $\tau_{\mu}$ & - & $R_{\mu v}(H)$ \\
space translations & $P_{a}$ & $E_{\mu}{ }^{a}$ & - & $R_{\mu v}{ }^{a}(P)$ \\
Galilean boosts & $G_{a}$ & $\Omega_{\mu}{ }^{a}$ & $\lambda^{a}\left(x^{v}\right)$ & $R_{\mu v}{ }^{a}(G)$ \\
spatial rotations & $J_{a b}$ & $\Omega_{\mu}{ }^{a b}$ & $\lambda^{a b}\left(x^{v}\right)$ & $R_{\mu \nu}{ }^{a b}(J)$ \\
central charge transf. & $Z$ & $M_{\mu}$ & $\sigma\left(x^{v}\right)$ & $R_{\mu v}(Z)$ \\
\hline
\end{tabular}

Table 1: This table indicates for every symmetry of the Bargmann algebra the corresponding generators, gauge fields, local gauge parameters and covariant curvatures.

with parameters $\xi^{\mu}(x)$. The two symmetries ( $H$ and $P$ transformations versus general coordinate transformations) are related to each other via a so-called trivial or Zilch symmetry [5].

One can now show that by imposing the 24 conventional constraints

$$
R_{\mu \nu}{ }^{a}(P)=R_{\mu v}(Z)=0
$$

the 24 connection fields $\Omega_{\mu}{ }^{a b}$ and $\Omega_{\mu}{ }^{a}$ can be solved in terms of independent gauge fiels . Furthermore, we impose the 6 geometric constraints

$$
R_{\mu v}(H)=2 \partial_{[\mu} \tau_{v]}=0 \quad \rightarrow \quad \tau_{\mu}=\partial_{\mu} \tau
$$

defining absolute time. Due to this constraint the time difference $\Delta T$ between two events becomes independent of the path $\mathscr{C}$ that the observer has traveled between these two events:

$$
\Delta T=\int_{\mathscr{C}} \mathrm{d} x^{\mu} \tau_{\mu}=\int_{\mathscr{C}} \mathrm{d} \tau
$$

One thus ends up with three independent gauge-fields $\left\{\tau_{\mu}, E_{\mu}{ }^{a}, M_{\mu}\right\}$ that transform under general coordinate transformations, with parameters $\xi^{\mu}$, as covariant vectors and under spatial rotations, Galilean boosts and central charge transformation as follows:

$$
\begin{aligned}
\delta \tau_{\mu} & =\xi^{\lambda} \partial_{\lambda} \tau_{\mu}+\partial_{\mu} \xi^{\lambda} \tau_{\lambda}, \\
\delta E_{\mu}{ }^{a} & =\xi^{\lambda} \partial_{\lambda} E_{\mu}{ }^{a}+\partial_{\mu} \xi^{\lambda} E_{\lambda}{ }^{a}+\lambda^{a}{ }_{b} E_{\mu}{ }^{b}+\lambda^{a} \tau_{\mu}, \\
\delta M_{\mu} & =\xi^{\lambda} \partial_{\lambda} M_{\mu}+\partial_{\mu} \xi^{\lambda} M_{\lambda}+\partial_{\mu} \sigma+\lambda_{a} E_{\mu}{ }^{a} .
\end{aligned}
$$

One may define the following Bargmann-invariant metrics

$$
\tau_{\mu v}=\tau_{\mu} \tau_{v}, \quad h^{\mu v}=E^{\mu}{ }_{a} E_{b}^{v} \delta^{a b}
$$

one in the time direction and a separate one in the spatial directions. One cannot define a boostinvariant metric with upper indices in the time direction or lower indices in the spatial directions. A Galilean boost-invariant metric can be obtained by adding terms proportional to the central charge gauge field $M_{\mu}$ as follows:

$$
H_{\mu v}=E_{\mu}^{a} E_{v}^{b} \delta_{a b}-2 \tau_{(\mu} M_{v)} .
$$


However, in that case one ends up with a metric that is not invariant under the central charge gauge transformation.

Newton-Cartan gravity can be obtained as a non-relativistic limit of general relativity, much in the same way as the Bargmann algebra can be obtained as the Inönü-Wigner contraction of the direct product of the Poincaré algebra and a U(1) generator. The latter has the physical interpretation that, when coupled to matter, its Noether charge corresponds to the conservation of particles minus anti-particles. To describe this particular Inönü-Wigner contraction we consider the Poincaré algebra plus an additional U(1) generator $\hat{\mathscr{Z}}$ that commutes with all the Poincaré generators:

$$
\left[\hat{P}_{\hat{A}}, \hat{M}_{\hat{B} \hat{C}}\right]=2 \eta_{\hat{A}[\hat{B}} \hat{P}_{\hat{C}]}, \quad\left[\hat{M}_{\hat{A} \hat{B}}, \hat{M}_{\hat{C} \hat{D}}\right]=4 \eta_{[\hat{A}[\hat{C}} \hat{M}_{\hat{D}] \hat{B}]} \text { plus } \quad \hat{Z} .
$$

Here $\left\{\hat{P}_{\hat{A}}, \hat{M}_{\hat{A} \hat{B}}\right\}$ are the generators of spacetime translations and Lorentz transformations, respectively. We have indicated all relativistic generators with a hat to distinguish them from the nonrelativistic case. The extra $\mathrm{U}(1)$ generator $\hat{\mathscr{Z}}$ is needed because the Bargmann algebra contains one generator more than the Poincaré algebra. Next, we decompose $\hat{A}=(0, a)$ and relate the Poincaré $\otimes \mathrm{U}(1)$ generators $\left\{\hat{P}_{0}, \hat{P}_{a} \hat{M}_{a 0}, \hat{M}_{a b}\right\}$ and $\hat{\mathcal{Z}}$ to the non-relativistic Bargmann generators $\left\{H, P_{a}, G_{a}, J_{a b}, Z\right\}$ as follows:

$$
\begin{aligned}
\hat{P}_{0} & =\frac{1}{2 \omega} H+\omega Z, & \hat{\mathcal{Z}} & =\frac{1}{2 \omega} H-\omega Z, \\
\hat{P}_{a} & =P_{a}, \quad \hat{M}_{a b}=J_{a b}, & \hat{M}_{a 0} & =\omega G_{a},
\end{aligned}
$$

where we have introduced a contraction parameter $\omega$. In a second step, taking the limit $\omega \rightarrow \infty$, we obtain the Bargmann algebra including the following commutator containing the central charge generator $Z$ :

$$
\begin{aligned}
& {\left[P_{A}, G_{B}\right]=\delta_{A B} Z} \\
& \text { Poincaré } \otimes \mathrm{U}(1) \quad \stackrel{\text { 'gauging' }}{\Longrightarrow} \quad \text { GR plus } \partial_{\mu} \hat{M}_{v}-\partial_{v} \hat{M}_{\mu}=0 \\
& \text { contraction } \Downarrow \quad \Downarrow \text { the NC limit } \\
& \text { Bargmann } \quad \stackrel{\text { gauging' }}{\Longrightarrow} \quad \text { Newton-Cartan gravity }
\end{aligned}
$$

Figure 1. This figure compares the Inönü-Wigner contraction of the Poincaré algebra times a U(1) with the non-relativistic limit of general relativity plus a zero flux gauge field $\hat{M}_{\mu}$ discussed in the text.

The non-relativistic limit of general relativity, which is inspired by the above Inönü-Wigner contraction, is introduced as follows, see Figure 1. Using a second-order formulation of general relativity, we first introduce, on top of the Vierbein field, a vector field $\hat{M}_{\mu}$ with $\partial_{[\mu} \hat{M}_{v]}=0$ [3]. Next, 
we relate the relativistic gauge fields $\left\{\hat{E}_{\mu}{ }^{\hat{A}}, \hat{M}_{\mu}\right\}$ to the non-relativistic gauge fields $\left\{\tau_{\mu}, E_{\mu}{ }^{a}, M_{\mu}\right\}$ of NC gravity as follows:

$$
\hat{E}_{\mu}{ }^{0}=\omega \tau_{\mu}+\frac{1}{2 \omega} M_{\mu}, \quad \hat{M}_{\mu}=\omega \tau_{\mu}-\frac{1}{2 \omega} M_{\mu}, \quad \hat{E}_{\mu}{ }^{a}=E_{\mu}{ }^{a} .
$$

In a second step we take the limit $\omega \rightarrow \infty$ in the relativistic transformation rules. In this way we obtain the correct non-relativistic transformation rules (2.5). The same limit can be used to derive the NC gravity equations of motion from the Einstein equations. Note that the standard textbooks on general relativity usually go straight from General Relativity to Newtonian gravity skipping the general frame formulation of Newtonian gravity.

A striking feature of the non-relativistic limit we just defined is that it requires the introduction of the auxiliary gauge field $\hat{M}_{\mu}$. Another way to see the necessity of this additional gauge field for taking the limit is considering the action of a particle moving in a general relativistic gravitational background. In order to define a limit without fatal divergencies one needs to couple the particle not only to general relativity but also to the gauge field $\hat{M}_{\mu}$ via a so-called Wess-Zumino term. This brings us to the remarkable conclusion that the limit of general relativity we just defined is particular to particles. It will not work for other extended objects such as strings. This leads to the following question:

\section{If NC gravity couples to particles what is the non - relativistic gravity that couples to strings?}

We will answer this question in the next section.

\section{String Newton-Cartan Gravity}

A basic difference between particles and strings is that a particle naturally couples to a 1-form gauge field $\hat{M}_{\mu}$ whereas a string couples to a 2 -form gauge field $\hat{M}_{\mu \nu}$. In order to cancel the leading divergence that occurs in the particle limit one needs to add a Wess-Zumino term to the particle kinetic term with

$$
\hat{M}_{\mu}=\omega \tau_{\mu}+\cdots
$$

In the case of strings the cancellation of the leading divergence requires that the 2-form gauge field $\hat{M}_{\mu \nu}$ starts as $\omega^{2}$ times something quadratic in $\tau_{\mu}$ but, due to the anti-symmetry of the 2-form, one cannot write down such an expression. One way to avoid this problem is to introduce two Vierbeine $\tau_{\mu}{ }^{A}$ where $A=0,1$ refer to the two (timelike and spacelike) directions longitudinal to the string. One writes

$$
\hat{M}_{\mu \nu}=\omega^{2} \tau_{\mu}^{A} \tau_{\nu}{ }^{B} \varepsilon_{A B}+\mathscr{O}\left(\omega^{0}\right) .
$$

The $\tau_{\mu}{ }^{A}$ are two generalized clock functions that define a so-called string NC geometry. The basic difference with the particle case is that the relativistic index $\hat{A}$ is divided up differently in longitudinal directions $A$ and transverse directions $a$ as follows:

particle: $\hat{A}=(0, a)$ with $a=1, \cdots, d$

string: $\quad \hat{A}=(A, a)$ with $A=0,1$ and $a=2, \cdots, d-1$. 
The string Galilei symmetries underlying this geometry are given by

$$
\begin{aligned}
\text { longitudinal translations } & H_{A} \\
\text { transverse translations } & P_{a} \\
\text { string Galilei boosts } & G_{A b} \\
\text { longitudinal Lorentz rotations } & M_{A B} \\
\text { transverse spatial rotations } & J_{a b}
\end{aligned}
$$

In gauging the algebra corresponding to these symmetries one again encounters extensions that in this case are non-central:

$$
\left[G_{A a}, P_{b}\right]=0 \quad \rightarrow \quad\left[G_{A a}, P_{b}\right]=\delta_{a b} Z_{A}
$$

and leads to non-central charge gauge fields $M_{\mu}{ }^{A}$. To obtain irreducibility one needs to impose a set of conventional and geometric constraints. A difference with the particle case is that the zero torsion constraint now becomes a mixture of conventional and geometric constraint:

$$
R_{\mu \nu}{ }^{A}(H)=D_{[\mu}(\Omega) \tau_{v]}^{A}=0
$$

For instance, in 4 spacetime dimensions, i.e. $d=3$, the 12 curvature constraints (3.7) is a mixture of 4 conventional constraint that can be used to solve for the 4 components of longitudinal spinconnection fields

$$
\Omega_{\mu}^{A B}=\varepsilon^{A B} \Sigma_{\mu}
$$

and 8 geometric constraints.

After gauging, one ends up with the independent string NC gauge fields $\left\{\tau_{\mu}{ }^{A}, E_{\mu}{ }^{a}, m_{\mu}{ }^{A}\right\}$ that transform under the different symmetries as follows:

$$
\begin{aligned}
\delta \tau_{\mu}{ }^{A} & =\Lambda^{A}{ }_{B} \tau_{\mu}{ }^{B}, \\
\delta E_{\mu}{ }^{a} & =\Lambda^{a}{ }_{b} E_{\mu}{ }^{b}-\Sigma_{A}{ }^{a} \tau_{\mu}{ }^{A}, \\
\delta m_{\mu}{ }^{A} & =D_{\mu} \sigma^{A}+\Sigma^{A}{ }_{a} E_{\mu}{ }^{a} .
\end{aligned}
$$

Furthermore, all gauge fields transform as covariant vectors under general coordinate transformations. These transformations rules are the generalization of the particle transformation rules (2.5) to strings and will play a prominent role as the symmetries on the non-relativistic Polyakov string we discuss in the next section.

We note that the string analogue of the longitudinal metric (2.6) and boost invariant metric (2.7) are given by

$$
\begin{aligned}
\text { longitudinal metric : } & \tau_{\mu \nu} \equiv \tau_{\mu}{ }^{A} \tau_{v}{ }^{B} \eta_{A B}, \\
\text { boost invariant metric: } & H_{\mu \nu} \equiv E_{\mu}{ }^{a} E_{v}{ }^{b} \delta_{a b}+\left(\tau_{\mu}{ }^{A} m_{v}{ }^{B}+\tau_{v}{ }^{A} m_{\mu}{ }^{B}\right) \eta_{A B} .
\end{aligned}
$$

Like in the particle case, $H_{\mu \nu}$ is invariant under string Galilei boost transformations but transforms under the non-central charge gauge transformations with parameter $\sigma^{A}$. 


\section{The Non-relativistic Polyakov String}

To understand the non-relativistic Polyakov string as the limit of the relativistic Polyakov string, it is instructive to first consider the particle case. Our starting point is a particle in a Polyakov formulation moving in the fields of general relativity and coupled to the extra gauge field $\hat{M}_{\mu}$ via a Wess-Zumino (WZ) term:

$$
S_{\text {Pol. }}=-\frac{1}{2} \int d \tau\left\{-\frac{1}{e} \hat{E}_{\mu}{ }^{\hat{A}} \dot{x}^{\mu} \hat{E}_{v} \hat{B}^{v} \eta_{\hat{A} \hat{B}}+M^{2} e-2 M \hat{M}_{\mu} \dot{x}^{\mu}\right\} .
$$

Here $e$ is the Einbein and $M$ a mass parameter. Substituing the parametrization (2.12) we obtain a potential leading divergence of order $c^{2}$ :

$$
S_{\text {Pol. }}\left(c^{2}\right)=-\frac{1}{2} c^{2} \int d \tau \frac{1}{e}\left[\tau_{\mu} \dot{x}^{\mu}-m e\right]^{2} .
$$

To circumvent this potential divergence, we introduce by hand a Lagrange multiplier $\lambda$ and rewrite the above term in the following equivalent form:

$$
S_{\text {Pol. }}\left(c^{2}\right)=-\frac{1}{2} \int d \tau \frac{1}{e}\left\{\lambda\left(\tau_{\mu} \dot{x}^{\mu}-m e\right)-\frac{1}{4 c^{2}} \lambda^{2}\right\} .
$$

Solving for $\lambda$ by using it's equation of motion one recovers the Lagrangian (4.2). The advantage of the Lagrangian (4.10) is that the quadratic divergence has disappeared. Note that this rewriting can only be done after making the expansion (2.12). Strictly speaking we can not consider the result we obtain as the limit of the original Polyakov Lagrangian (4.1).

Continuing in this way, we obtain the following Lagrangian for the non-relativistic Poyakov particle:

$$
S_{\text {Pol. }}(\text { N.R. })=-\frac{1}{2} \int d \tau \frac{1}{e}\left\{\dot{x}^{\mu} \dot{x}^{v} H_{\mu v}+\lambda\left(\tau_{\mu} \dot{x}^{\mu}-m e\right)\right\}
$$

Note that the boost invariant metric $H_{\mu \nu}$ defined in eq. (2.7) occurs in the Lagrangian. The fact that this metric is not invariant under the central charge transformation leads to a total derivative in the Lagrangian. Solving for $e$ by using the equation of motion for $\lambda$ leads to the Lagrangian for the non-relativistic Nambu-Goto particle. Note that the resulting Nambu-Goto Lagrangian is non-linear in the longitudinal time coordinate $\tau_{\mu} \dot{x}^{\mu}$ but quadratic in the transverse coordinates that are contracted with the boost invariant metric $H_{\mu \nu}$. This is in contrast to the Lagrangian for the relativistic Nambu-Goto particle which is non-linear in all directions.

The Lagrangian (4.4) for the non-relativistic Polyakov particle has all the features of the nonrelativistic Polyakov string. To derive the action for the non-relativistic Polyakov string, the relativistic gauge fields are redefined as follows;

$$
\begin{array}{ll}
\hat{E}_{\mu}{ }^{A}=\omega \tau_{\mu}{ }^{A}+\frac{1}{\omega} m_{\mu}{ }^{A}, & \hat{E}_{\mu}{ }^{a}=E_{\mu}{ }^{a}, \\
\hat{M}_{\mu \nu} & =\omega^{2} \tau_{\mu}{ }^{A} \tau_{\nu}{ }^{B} \varepsilon_{A B}+B_{\mu \nu} .
\end{array}
$$

and two Lagrange multipliers $\lambda$ and $\bar{\lambda}$ are introduced to avoid divergences when the limit is taken. We have used here the following definitions. First of all, we have defined a worldsheet metric $h_{\alpha \beta}$ that can be expressed in terms of worldsheet Zweibeine as follows:

$$
h_{\alpha \beta}=e_{\alpha}^{a} e_{\beta}^{b} \eta_{a b}
$$


with $\alpha=0,1(a=0,1)$ curved (flat) worldsheet indices. ${ }^{2}$ Furthermore, for $e_{\mu}{ }^{a}$ and $\tau_{\mu}{ }^{A}$ we use the following lightcone notation:

$$
\begin{aligned}
e_{\alpha} \equiv e_{\alpha}{ }^{0}+e_{\alpha}{ }^{1}, & \bar{e}_{\alpha} \equiv e_{\alpha}{ }^{0}-e_{\alpha}{ }^{1}, \\
\tau_{\mu} \equiv \tau_{\mu}{ }^{0}+\tau_{\mu}{ }^{1}, & \bar{\tau}_{\mu} \equiv \tau_{\mu}{ }^{0}-\tau_{\mu}{ }^{1} .
\end{aligned}
$$

On top of definitions above, by taking $\omega \rightarrow \infty$ limit of relativistic Polyakov action of a string that is coupled to both the target space metric $\hat{G}_{\mu \nu}$ and 2-form gauge field $\hat{M}_{\mu \nu}$, the non-relativistic Polyakov string action

$$
\begin{aligned}
S_{\text {Pol. }}= & -\frac{T}{2} \int d^{2} \sigma\left[\sqrt{-h} h^{\alpha \beta} \partial_{\alpha} x^{\mu} \partial_{\beta} x^{v} H_{\mu v}+\varepsilon^{\alpha \beta}\left(\lambda e_{\alpha} \tau_{\mu}+\bar{\lambda}_{\bar{e}_{\alpha}} \bar{\tau}_{\mu}\right) \partial_{\beta} x^{\mu}\right] \\
& -\frac{T}{2} \int d^{2} \sigma \varepsilon^{\alpha \beta} \partial_{\alpha} x^{\mu} \partial_{\beta} x^{v} B_{\mu \nu}
\end{aligned}
$$

is obtained. Using the equations of motion of the Lagrange multipliers $\lambda$ and $\bar{\lambda}$ one is able to solve for the worldsheet metric $h_{\mu v}$ in terms of the longitudinal metric $\tau_{\mu v}$ up to a scale factor:

$$
h_{\alpha \beta} \sim \partial_{\alpha} x^{\mu} \partial_{\beta} x^{v} \tau_{\mu v}
$$

Substituting this solution back into the non-relativistic Polyakov Lagrangian (4.10) one obtains the Nambu-Goto formulation of the non-relativistic Polyakov string [14].

The non-relativistic Polyakov string defined by the Lagrangian (4.10) is a central result. The nice thing is that, although it was obtained as a kind of limit of the relativistic Polyakov string, one could use the Lagrangian (4.10) as an independent starting point to investigate the properties of non-relativitic string theory. Imposing the worldsheet conformal gauge

$$
\sqrt{-h} h^{\alpha \beta}=\eta^{\alpha \beta}
$$

and taking the flat spacetime background

$$
\tau_{\mu}^{A}=\delta_{\mu}^{A}, \quad E_{\mu}{ }^{a}=\delta_{\mu}^{a}, \quad M_{\mu}{ }^{a}=0
$$

one recovers the Lagrangian of the non-relativistic string theory of [6]

$$
S=-\frac{T}{2} \int d^{2} \sigma\left(\partial x^{a} \bar{\partial} x^{b} \delta_{a b}+\lambda \bar{\partial} X+\bar{\lambda} \partial \bar{X}\right) .
$$

where

$$
\begin{aligned}
X=x_{0}+x_{1} & \bar{X}=x_{0}-x_{1}, \\
\partial=\partial_{0}+\partial_{1} & \bar{\partial}=-\partial_{0}+\partial_{1} .
\end{aligned}
$$

We have generalized this Lagrangian to a string moving in an arbitrary non-relativistic gravitational background and, moreover, we have identified the geometry underlying the non-relativistic string

\footnotetext{
${ }^{2}$ Note that we use the index $a$ both for flat worldsheet indices as well as for flat transverse target space indices. Whenever confusion could occur we will specify which index we mean.
} 
as the string $\mathrm{NC}$ geometry discussed in section 2 . We could now use this relation to study some of the properties of string $\mathrm{NC}$ geometry in the same way as we are doing in the relativistic case. One of these peculiar properties is the behaviour of the geometry under the so-called T-duality tranformations. In the next section we will derive the non-relativistic T-duality properties using the non-relativistic Polyakov Lagrangian (4.10) as our starting point. The non-relativistic limit introduced above is also applied to the Eintein-Hilbert action plus a term contains an auxiliary twoform and one-form gauge field and it produces the extended string Newton-Cartan gravity[11].

\section{T-duality}

Before discussing the non-relativistic case, it is instructive to first go shortly over the relativistic case. Following $[8,9,10]$, we assume that the geometry has a spacelike Killing vector $k^{\mu}$. Using adapted coordinates with isometry direction $y$

$$
x^{\mu}=\left(y, x^{i}\right) \quad k^{\mu} \partial_{\mu}=\partial_{y}
$$

we rewrite the non-relativistic Polyakov Lagrangian (4.10) as the following parent action:

$$
S_{\text {parent }}=\underbrace{S_{\text {Pol. }}\left(\partial_{\alpha} y \rightarrow v_{\alpha}\right)}_{\text {quadratic in } v_{\alpha} !}-T \int d^{2} \sigma \varepsilon^{\alpha \beta} \tilde{y} \partial_{\alpha} v_{\beta},
$$

where we have replaced everywhere $\partial_{\alpha} y$ by a new field $v_{\alpha}$ and, moreover, have introduced a new Lagrange multiplier field $\tilde{y}$ who in a minute is going to play the role of the dual coordinate. The important point is that the Polyakov Lagrangian (4.10) is quadratic in $\partial y$ and, hence, the parent action as given in (5.2) is quadratic in $v_{\alpha}$.

Starting from the parent action (5.2) we can follow two approaches. One, we impose the equation of motion corresponding to the Lagrange multiplier $\tilde{y}$ leading to the Bianchi identiy for $v_{\alpha}$ whose solution is given by

$$
v_{\alpha}=\partial_{\alpha} y
$$

Substituting this solution back into the action corresponding to the Polyakov Lagrangian (4.10) one re-obtains the Polyakov action we started from. Instead of doing this, we can also solve for the equation of motion of $v_{\alpha}$. Since the first term in the parent action (5.2) is quadratic in $v_{\alpha}$ this leads to the following solution for $v_{\alpha}$ :

$$
v_{\alpha}=\varepsilon_{\alpha}^{\beta} \partial_{\beta} \tilde{y} .
$$

Substituting this solution back into the parent action (5.2) leads in the relativistic case to a Polyakov string moving in a dual geometric background. For instance, taking the ten-dimensional background to be a nine-dimensional Minkowski spacetime times a circle with radius $R$, after the duality transformation one ends up with the same geometry but now with $\mathrm{R}$ replaced by $1 / R$ :

$$
R \quad \stackrel{T}{\rightarrow} 1 / R
$$

This is a special case of the so-called Buscher rules $[8,9,10]$.

We now wish to perform the same manipulations for the non-relativistic Polyakov string and derive the non-relativistic version of the Buscher rules. First of all, it matters whether we dualise 
in the longitudinal or transverse direction. This has to do with the fact that the term with the extra Lagrange multipliers $\lambda$ and $\bar{\lambda}$ only depends on the longitudinal embedding coordinates. These extra terms, which are absent in the relativistic case, can therefore only effect the longitudinal T-duality transformations. Following [7] we find the following results:

(i) The longitudinal spatial T-dual of the NR string is the Polyakov string moving in a general relativity background with a lightlike direction.

(ii) The transverse spatial T-dual of the NR string is again a NR string but with a transverse spatial isometry direction given by the Buscher rules, i.e. a transverse circle with radius $R$ becomes a transverse circle with radius $1 / R$.

\section{Conclusions}

In this talk we showed that the geometry underlying non-relativistic string theory is given by the string NC geometry as discussed in section 2 . In section 4 we derived the action describing a non-relativistic Polyakov string moving in this string NC background. This led to the action (4.10) which should be the starting point for every effort to study non-relativistic string theory. As an example, we showed in this talk how the action (4.10) could be used to obtain the non-relativistic T-duality rules which gives information on how a non-relativistic string feels it's own geometry.

There are many other things one can study now, like, e.g., the calculation of the non-relativistic beta functions which would determine the consistent backgrounds in which the non-relativistic string can move. Our hope is that at the end we may use non-relativistic string theory as defined by the Lagrangian (4.10) to define non-relativistic holography and in this way learn something about the (non-perturbative) properties of non-relativistic conformal quantum field theories with (infinitedimensional) Galilean conformal symmetries that could not be studied using general relativity in the bulk.

\section{Acknowledgements}

This talk was based upon the papers [7, 11, 12]. We thank Jaume Gomis, K. T. Grosvenor, Jan Rosseel, Thomas Zojer for the many stimulating discussions we had with them. We also thank the organizers for a very stimulating atmosphere.

\section{References}

[1] A. Bagchi and R. Gopakumar, Galilean Conformal Algebras and AdS/CFT, JHEP 0907 (2009) 037 doi:10.1088/1126-6708/2009/07/037 [arXiv:0902.1385 [hep-th] ].

[2] D. T. Son and M. Wingate, General coordinate invariance and conformal invariance in nonrelativistic physics: Unitary Fermi gas, Annals Phys. 321 (2006) 197 doi:10.1016/j.aop.2005.11.001; C. Hoyos and D. T. Son, Hall Viscosity and Electromagnetic Response, Phys. Rev. Lett. 108, 066805 (2012) doi:10.1103/PhysRevLett.108.066805; D. T. Son, Newton-Cartan Geometry and the Quantum Hall Effect. 
[3] E. Bergshoeff, J. Rosseel and T. Zojer, Non-relativistic fields from arbitrary contracting backgrounds, Class. Quant. Grav. 33 (2016) no.17, 175010 doi:10.1088/0264-9381/33/17/175010 [arXiv:1512.06064 [hep-th]].

[4] R. Andringa, E. Bergshoeff, S. Panda and M. de Roo, Newtonian Gravity and the Bargmann Algebra, Class. Quant. Grav. 28 (2011) 105011 doi:10.1088/0264-9381/28/10/105011 [arXiv: 1011.1145 [hep-th]].

[5] D. Z. Freedman and A. Van Proeyen, Supergravity, Cambridge University Press (2012).

[6] J. Gomis and H. Ooguri, Nonrelativistic closed string theory, J. Math. Phys. 42 (2001) 3127 doi:10.1063/1.1372697 [hep-th/0009181].

[7] E. Bergshoeff, J. Gomis and Z. Yan, Nonrelativistic String Theory and T-Duality, JHEP 1811 (2018) 133 doi:10.1007/JHEP11(2018)133 [arXiv:1806.06071 [hep-th]].

[8] T. H. Buscher, A Symmetry of the String Background Field Equations, Phys. Lett. B 194 (1987) 59. doi:10.1016/0370-2693(87)90769-6

[9] T. H. Buscher, Path Integral Derivation of Quantum Duality in Nonlinear Sigma Models, Phys. Lett. B 201 (1988) 466. doi:10.1016/0370-2693(88)90602-8

[10] M. Rocek and E. P. Verlinde, Duality, quotients, and currents, Nucl. Phys. B 373 (1992) 630 doi:10.1016/0550-3213(92)90269-H [hep-th/9110053].

[11] E. A. Bergshoeff, K. T. Grosvenor, C. Simsek and Z. Yan, An Action for Extended String Newton-Cartan Gravity, JHEP 1901 (2019) 178 doi:10.1007/JHEP01(2019)178 [arXiv:1810.09387 [hep-th]].

[12] E. Bergshoeff, J. Rosseel and T. Zojer, Newton-Cartan (super)gravity as a non-relativistic limit, Class. Quant. Grav. 32 (2015) no.20, 205003 doi:10.1088/0264-9381/32/20/205003 [arXiv:1505.02095 [hep-th]].

[13] M. H. Christensen, J. Hartong, N. A. Obers and B. Rollier, Torsional Newton-Cartan Geometry and Lifshitz. Holography, Phys. Rev. D 89 (2014) 061901 doi:10.1103/PhysRevD.89.061901 [arXiv:1311.4794 [hep-th]].

[14] R. Andringa, E. Bergshoeff, J. Gomis and M. de Roo, 'Stringy' Newton-Cartan Gravity, Class. Quant. Grav. 29 (2012) 235020 doi:10.1088/0264-9381/29/23/235020 [arXiv:1206.5176 [hep-th] ]. 